\title{
Mixed Convection Flow of Non-Newtonian Fluid from Vertical Surface Saturated in a Porous Medium Filled with a Nanofluid
}

\author{
A. M. $\operatorname{Rashad}^{1 \dagger}$, A. J. Chamkha ${ }^{2}$ and M. M. M. Abdou ${ }^{1}$ \\ ${ }^{1}$ Department of Mathematics, Aswan University, Aswan, Egypt \\ ${ }^{2}$ Manufacturing Engineering Department, The Public Authority for Applied Education and Training, Shuweikh \\ 70654, Kuwait
}

†Corresponding Author Email: am_rashad@yahoo.com

(Received September 15, 2011; accepted May 21, 2012)

\begin{abstract}
The problem of steady, laminar, mixed convection flow of a non-Newtonian fluid past a preamble vertical flat plate embedded in a porous medium saturated with a nanofluid is considered. A mixed convection parameter for the entire range of free-forced-mixed convection is employed and a set of non-similar equations are obtained. These equations are solved numerically by an efficient implicit, iterative, finite-difference method. The obtained results are checked against previously published work for special cases of the problem and are found to be in good agreement. A parametric study illustrating the influence of the various physical parameters on the velocity, temperature and nanoparticle volume fraction profiles as well as the local Nusselt and Sherwood numbers is conducted. The obtained results are illustrated graphically and the physical aspects of the problem are discussed.
\end{abstract}

Keywords: Mixed convection, Porous medium, Non-Newtonian fluid, Nanofluid.

\section{NOMENCLATURE}

C nanoparticle volume fraction

$\mathrm{C}_{\mathrm{w}}$ nanoparticle volume fraction at the vertical plate

$\mathrm{C}_{\infty} \quad$ ambient nanoparticle volume fraction attained as y tends to infinity

$D_{B} \quad$ Brownian diffusion coefficient

$\mathrm{D}_{\mathrm{T}} \quad$ thermophoretic diffusion coefficient

$f$ dimensionless stream function

g gravitational acceleration vector

$\mathrm{K}$ permeability of porous edium

$\mathrm{k}_{\mathrm{f}} \quad$ thermal conductivity

Le Lewis number

$\mathrm{Nr}$ buoyancy ratio

$\mathrm{Nb}$ brownian motion parameter

$\mathrm{Nt}$ thermophoresis parameter

$\mathrm{n}$ power-law index

$\mathrm{Nu}_{\mathrm{x}} \quad$ local Nusselt number

$\mathrm{Pe}_{\mathrm{x}} \quad$ Peclet number

$\mathrm{Ra}_{\mathrm{x}} \quad$ local Rayleigh number

$\mathrm{Sh}_{\mathrm{x}} \quad$ local Sherwood number

$\mathrm{T}$ temperature

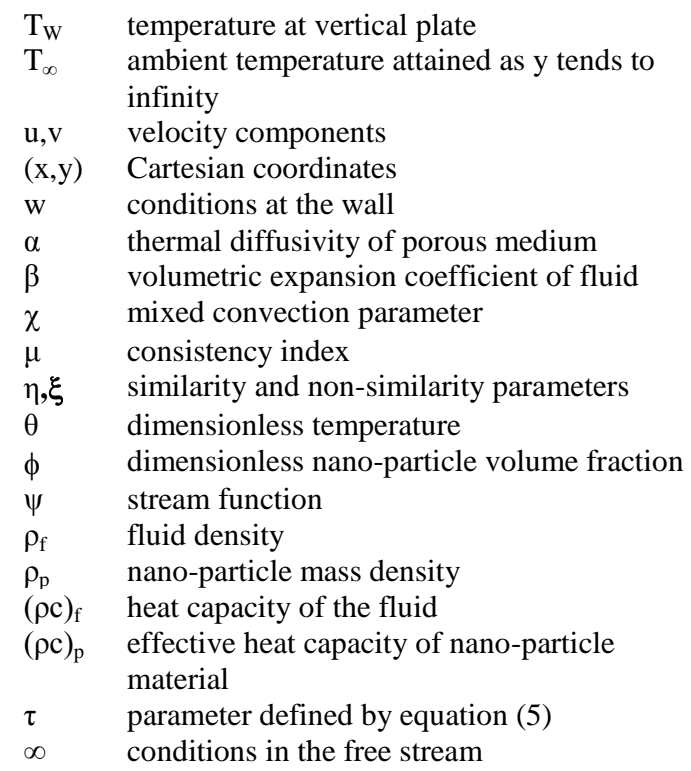




\section{INTRODUCTION}

The study of laminar boundary layer flow heat transfer in non-Newtonian fluids from surfaces through porous media has received considerable attention in recent years because it is an important type of scientific and engineering applications such as aerodynamic extrusion of plastic sheets and fibers, drawing, annealing and tinning of copper wire, paper production, crystal growing and glass blowing. Such applications involve cooling of a molten liquid by drawing it in to a cooling system. Chen and Chen (1988) presented similarity solutions for free convection of non-Newtonian fluids over vertical surfaces in porous media. Nakayama and Koyama (1991) studied the natural convection over a non-isothermal body of arbitrary shape embedded in a porous medium. Gorla et al. (1997) analyzed the mixed convection from a vertical plate in non-Newtonian fluid saturated porous media. Chiu et al. (1996) analyzed the effect of non-Newtonian fluids and porous media parameters on two-phase flow in porous media. Effect of porosity on the free convection flow of nonNewtonian fluids along vertical plate embedded in a porous media was presented by Beithou et al. (1998). Yih (1998) investigated the effect uniform lateral mass flux on natural convection of non-Newtonian fluids over a cone in porous media. Free convection heat transfer of non-Newtonian fluids over axisymmetric and two dimensional bodies of arbitrary shape embedded in a fluid-saturated porous media studied by Yang and Wang (1996). Pascal and Pascal (1997) studied the free convection in a non-Newtonian fluid saturated porous media with lateral mass flux. Gorla and Kumari (2000) obtained the non-similar solution for mixed convection in non-Newtonian fluids along a wedge with variable surface heat flux. The problem of coupled heat and mass transfer by mixed convective flow of a non- Newtonian power-law fluid over a permeable wedge embedded in a porous medium is considered by Chamkha (2010). EL-Kabeir et al. (2010) considered the coupled heat and mass transfer by mixed convection stagnation-point flow of a power-law nonNewtonian fluid towards a stretching surface in the presence of thermal-diffusion and diffusion-thermo effects.

On other hand, Nanotechnology has been widely used in industry since materials with sizes of nanometers possess unique physical and chemical properties. Nanoscale particle added fluids are called as nanofluid which is firstly utilized by Choi (1995). These fluids represent an innovative way to increase thermal conductivity and, therefore, heat transfer. Unlike heat transfer in conventional fluids, the exceptionally high thermal conductivity of nanofluids provides for exceptional heat transfer, a unique feature of nanofluids. Advances in device miniaturization have necessitated heat transfer systems that are small in size, light mass, and highperformance. The utility of a particular nanofluid for a heat transfer application can be established by suitably modeling the convective transport in the nanofluid, see (Kumar et al. 2009). Because the prospect of nanofluids is very promising, several studies of convective heat transfer in nanofluids have been reported in recent years. Xuan and Roetzel (2000) proposed homogeneous flow model where the convective transport equations of pure fluids are directly extended to nanofluids. This means that all traditional heat transfer correlations (e.g. Dittus-Boelter) could be used for nanofluids provided the properties of pure fluids are replaced by those of nanofluids involving the volume fraction of the nanoparticles. The homogeneous flow models are however in conflict with the experimental observations of Maliga et al. (2005), as they under predict the heat transfer coefficient of nanofluids. Kang et al. (2006) studied estimation of thermal conductivity of nanofluid using experimental effective particle volume. Application of nanofluids for heat transfer enhancement of separated flows encountered in a backward facing step presented by Abu-Nada (2008). Maiga et al. (2005) analyzed the heat transfer enhancement by using nanofluids in forced convection flows. Numerical research of nature convective heat transfer enhancement filled with nanofluids in rectangular enclosures proposed by Jou and Tzeng (2006). Hwang et al. (2007) reported a buoyancy-driven heat transfer of water-based on nanofluids in a rectangular cavity. Numerical study of natural convection in partially heated rectangular enclosures filled with nanofluids conducted by Tiwari and Das (2007). Oztop and Abu-Nada (2008) investigated a numerical study of natural convection in partially heated rectangular enclosures filled with nanofluids. Effects of inclination angle on natural convection in enclosures filled with $\mathrm{Cu}$-water nanofluid studied by Abu-Nada and Oztop (2009). Duangthongsuk and Wongwises (2008) analyzed the effect of thermophysical properties models on the predicting of the convective heat transfer coefficient for low concentration nanofluid. Chamkha et al. $(2010$, a) studied the mixed convection MHD flow of a nanofluid past a stretching permeable surface in the presence of magnetic field, heat generation or absorption, thermopherosis, Brownian motion and suction or injection effects. Chamkha et al. (2010 b) has also analyzed the natural convection past a sphere embedded in a porous medium saturated by a nanofluid.

The objective of the present study is to analyze the development of mixed convection flow of nonNewtonian nanofluid from vertical surface saturated in a porous medium. The effects of Brownian motion and thermophoresis are included for the nanofluid. Numerical solutions of the boundary layer equations are obtained and discussion is provided for several values of the nanofluid parameters governing the problem. The dependency of velocity, temperature and nano-particle volume fraction profiles as well as the surface heat transfer rate (local Nusselt number) and mass transfer rate (local Sherwood number) on these parameters has been discussed.

\section{ANALysis}

Consider steady, laminar, mixed convection, boundary layer flow of a non-Newtonian power-law fluid from a permeable vertical plate embedded in a nano-fluid saturated porous medium in the presence of Brownian motion, thermophoresis and injection effects. The plate surface is maintained at a constant temperature $T_{w}$ and a constant nano-particle volume fraction $C_{w}$, and the 
ambient temperature and nano-particle volume fraction far away from the surface $T_{\infty}$ and $C_{\infty}$ are assumed to be uniform. The fluid properties are considered to be constant. By invoking all of the boundary layer, Oberbeck-Boussineq approximations, the governing equations for this investigation can be written as:

$$
\begin{aligned}
& \frac{\partial u}{\partial x}+\frac{\partial v}{\partial y}=0 \\
& \frac{\partial u^{n}}{\partial y}=\frac{\left(1-C_{\infty}\right) \rho_{f \infty} \beta g K}{\mu} \frac{\partial T}{\partial y} \\
& u \frac{\partial T}{\partial x}+v \frac{\partial T}{\partial y}=\alpha \frac{\partial^{2} T}{\partial y^{2}} \\
& +\frac{\left(\rho_{P}-\rho_{f \infty}\right) g K}{\mu C} \frac{\partial C}{\partial y} \\
& \frac{1}{\varepsilon}\left(u \frac{\partial C}{\partial x}+\frac{\partial T}{\partial y}+\frac{D_{T}}{D_{\infty}}\left(\frac{\partial T}{\partial y}\right)^{2}\right]=D_{B} \frac{\partial^{2} C}{\partial y^{2}}+\left(\frac{D_{T}}{T_{\infty}}\right) \frac{\partial^{2} T}{\partial y^{2}}
\end{aligned}
$$

where

$$
\alpha=\frac{k}{(\rho c)_{f}}, \quad \tau=\frac{\varepsilon(\rho c)_{p}}{(\rho c)_{f}}
$$

The associated boundary conditions are as follows:

$$
\begin{aligned}
& v=v_{0}, \quad T=T_{W}, \quad C=C_{W}, \quad \text { at } y=0 \\
& u \rightarrow u_{\infty}, T \rightarrow T_{\infty}, C \rightarrow C_{\infty}, \text { as } y \rightarrow \infty
\end{aligned}
$$

It is convenient to transform Eq. (1) to Eq. (4) by using the following non-similarity transformations reported earlier by Gorla et al. (1997, 2000):

$$
\begin{aligned}
& \eta=\frac{y}{x}\left\{\sqrt{P e_{x}}+\sqrt{R a_{x}}\right\} \\
& \xi=\frac{v 0 x}{\alpha}\left\{\sqrt{P e_{x}}+\sqrt{R a_{x}}\right\}^{-1} \\
& P e_{x}=u_{\infty} x / \alpha \\
& \psi=\alpha\left\{\sqrt{P e_{x}}+\sqrt{R a_{x}}\right\} f(\xi, \eta) \\
& R a_{x}=(x / \alpha)\left\{\left(1-C_{\infty}\right) \rho_{f} g \beta \mathrm{K}\left(T_{w}-T_{\infty}\right) / \mu\right\}_{n} \\
& \theta=\frac{T-T}{T-T} \\
& \phi=\frac{C-C_{\infty}}{C_{w}-C_{\infty}}
\end{aligned}
$$

where $\mathrm{Pe}_{\mathrm{x}}$ and $\mathrm{Ra}_{\mathrm{x}}$ are the local Peclet and modified Rayleigh numbers, respectively, and $\psi$ is the stream function defined as $u=\partial \psi / \partial y$ and $v=-\partial \psi / \partial x$, therefore the continuity equation is identically satisfied.
Substituting Eq. (7) into Eq. (1)- (4) yield the following non-similar equations and boundary conditions:

$$
\begin{gathered}
n f^{\prime n-1} f^{\prime \prime}=(1-\chi)^{2 n}\left(\theta^{\prime}-N r \phi^{\prime}\right) \\
\theta^{\prime \prime}+N b \phi^{\prime} \theta^{\prime}+\frac{1}{2} f \theta^{\prime}+N t \theta^{\prime 2} \\
=\frac{1}{2} \xi\left(f^{\prime} \frac{\partial \theta}{\partial \xi}-\theta^{\prime} \frac{\partial f}{\partial \xi}\right) \\
\phi^{\prime \prime}+\frac{L e}{2} f \phi^{\prime}+\frac{N t}{N b} \theta^{\prime \prime} \\
=\frac{L e}{2} \xi\left(f^{\prime} \frac{\partial \phi}{\partial \xi}-\phi^{\prime} \frac{\partial f}{\partial \xi}\right)
\end{gathered}
$$

The dimensionless boundary conditions become:

$$
\begin{aligned}
& f(\xi, \eta)+\xi \frac{\partial}{\partial \xi} f(\xi, \eta)=-2 \xi, \\
& \theta(\xi, \eta)=\phi(\xi, \eta)=1 \text { as } \eta=0 \\
& f^{\prime}(\xi, \eta)=\chi^{2},
\end{aligned}
$$$$
\theta(\xi, \eta), \phi(\xi, \eta) \rightarrow 0 \text { as } \eta \rightarrow \infty
$$

where a prime denotes partial differentiation with respect to $\eta$ and

$$
\begin{aligned}
& \chi=\left[1+\sqrt{R a_{x} / P e_{x}}\right]^{-1} \\
& L e=\alpha / D_{B} \\
& N r=\frac{\left(\rho_{p}-\rho_{f \infty}\right)\left(C_{w}-C_{\infty}\right)}{\rho_{f \infty} \beta\left(T_{w}-T_{\infty}\right)\left(1-C_{\infty}\right)} \\
& N b=\frac{\varepsilon(\rho c)_{p} D_{B}\left(C_{w}-C_{\infty}\right)}{(\rho c)_{f} \alpha} \\
& N t=\frac{\varepsilon(\rho c)_{p} D_{T}\left(T_{w}-T_{\infty}\right)}{(\rho c)_{f} \alpha T_{\infty}}
\end{aligned}
$$

are the mixed convection parameter, Lewis number, buoyancy ratio, Brownian motion parameter and thermophoresis parameter. It can be noted that $\chi=0$ corresponds to pure natural convection whereas $\chi=1$ corresponds to pure forced convection. $\xi$ is positive for injection and negative for suction. The local Nusselt number $N u_{x}$ and the local Sherwood number $S h_{x}$ are important physical properties. These can be defined in dimensionless form below as:

$\frac{N u_{x}}{\sqrt{P e_{x}}+\sqrt{R a_{x}}}=-\theta^{\prime}(\xi, 0)$

$\frac{S h_{x}}{\sqrt{P e_{x}}+\sqrt{R a_{x}}}=-\phi^{\prime}(\xi, 0)$ 


\section{NUMERICAL METHOD}

The problem represented by Eq. (8) through Eq. (10) is nonlinear and has no closed-form solution. Therefore, it must be solved numerically. The implicit, tri-diagonal, finite-difference method discussed by Blottner (1970) has proven to be adequate for the solution of boundarylayer equations accurately. For this reason, it is adopted in this work. All first-order derivatives with respect to $\xi$ are replaced by two-point backward difference quotients while the derivatives with respect to $\eta$ are discretized using three-point central-difference quotients and, as a consequence, a set of algebraic equations results at each line of constant $\xi$. These algebraic equations are then solved by the well-known Thomas algorithm (see Blottner 1970) with iteration to deal with the non- linearities of the problem. When the solution at a specific line of constant $\xi$ is obtained, the same solution procedure is used for the next line of constant $\xi$. This marching process continues until the desired value of $\xi$ is reached. At each line of constant $\xi$, when $\mathrm{f}^{\prime}$ is known, the trapezoidal rule is used to solve for $\mathrm{f}$. The convergence criterion employed was based on the relative difference between the current and the previous iterations. When this difference reached $10^{-5}$, the solution was assumed converged and the iteration procedure was terminated. Constant step sizes in the $\xi$ direction were used whereas variable step sizes in the $\eta$ direction were utilized in order to accommodate the sharp changes in the dependent variables especially in the immediate vicinity of the truncated cone surface. The $(\xi, \eta)$ computational domain consisted of 101 and 196 points, respectively. The initial step sizes in $\Delta \xi_{1}$ and $\Delta \eta_{1}$ were taken to be equal to $10^{-2}$ and $10^{-3}$, respectively and the growth factor for the $\eta$ direction was taken to be 1.0375 . This gave $\xi_{\infty}=1$ and $\eta_{\infty}=25$. These values were found to give accurate gridindependent results as verified by the comparisons mentioned below.

In order to access the accuracy of the numerical results, we have compared the results obtained by this numerical method with the previously published work of Gorla et al. (1997) for the pure natural convection $\chi=$ 0 to the pure forced convection $\chi=1$ with various values of $\mathrm{n}$ and $\xi$. This comparison is presented in Tables 1. It is obvious from these tables that excellent agreement between the results exist. These favourable comparisons lend confidence in the graphical results to be reported in the next section.

\section{DESCRIPTION OF REFERENCES}

In this section, a representative set of numerical results for the velocity, temperature, and nano-particle volume fraction profiles as well as the local Nusselt number and the local Sherwood number is presented graphically in Fig. 1 through Fig. 15. These results illustrate the effects of the power-law index parameter n, Lewis number, buoyancy ratio, Brownian motion parameter and thermophoresis parameter on the solutions. Throughout the calculations, these conditions are intended for pure natural convection $\chi=0$ pure forced convection $\chi=1$ with positive values of $\xi$ (injection case).
Table 1 Comparison of the surface heat transfer rate $-\theta^{\prime}(\xi, 0)$ in the entire range of mixed convection for various values of $\xi$ and $n$ in the absence of mass transfer

\begin{tabular}{cccc}
\hline \multicolumn{4}{c}{ Present results } \\
\hline$\xi$ & 0.0 & 0.5 & 1.0 \\
$\mathrm{n}=0.5$ & & & \\
$\chi=0.0$ & 0.50937 & 0.18075 & 0.03122 \\
$\chi=0.5$ & 0.53464 & 0.149696 & 0.03556 \\
$\chi=1.0$ & 0.564129 & 0.198932 & 0.047507 \\
$\mathrm{n}=1.5$ & & & \\
$\chi=0.5$ & 0.3607312 & 0.07037 & 0.00665 \\
$\mathrm{n}=2.0$ & & & \\
$\chi=0.5$ & 0.3177039 & 0.04429 & 0.00235 \\
\hline & Gorla et & al. $(1997)$ & \\
\hline$\xi$ & 0.0 & 0.5 & 1.0 \\
$\mathrm{n}=0.5$ & & & 0.03121 \\
$\chi=0.0$ & 0.50935 & 0.18076 & 0.0023 \\
$\chi=0.5$ & 0.5348 & 0.149699 & 0.03383 \\
$\chi=1.0$ & 0.5642 & 0.19894 & 0.04753 \\
$\mathrm{n}=1.5$ & & & \\
$\chi=0.5$ & 0.3603 & 0.0704 & 0.0069 \\
$\mathrm{n}=2.0$ & & & \\
$\chi=0.5$ & 0.3184 & 0.04419 & 0.003 \\
\hline
\end{tabular}

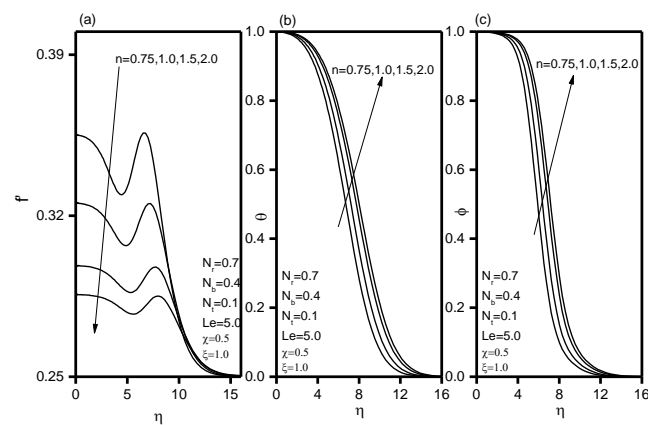

Fig. 1. Effects of $n$ on the (a) velocity, (b) temperature, (c) volume fraction profiles

Figures 1(a)- 1(c) present the typical velocity $f^{\prime}$, temperature $\theta$ and volume fraction $\phi$ profiles for various values of the non-Newtonian fluid (viscosity index) $n$. for power-law fluid viscosity indices $n=0.75$ (shear-thinning or pseudo-plastic fluid), $\mathrm{n}=1.0$ (Newtonian fluid) and $n=1.5,2.0$ (shear-thickening or dilatant fluid), respectively. It is observed that as the power-law index $\mathrm{n}$ increases, both the volume fraction and temperature increase, while the slip velocity at the 
preamble wall decreases. In addition, the nonNewtonian fluid with a higher power-law index has a greater thermal boundary layer thickness and a greater volume fraction boundary-layer thickness. Moreover, increasing the power-law index tends to retard the flow and increase the thermal boundary-layer and the volume fraction boundary layer thicknesses.

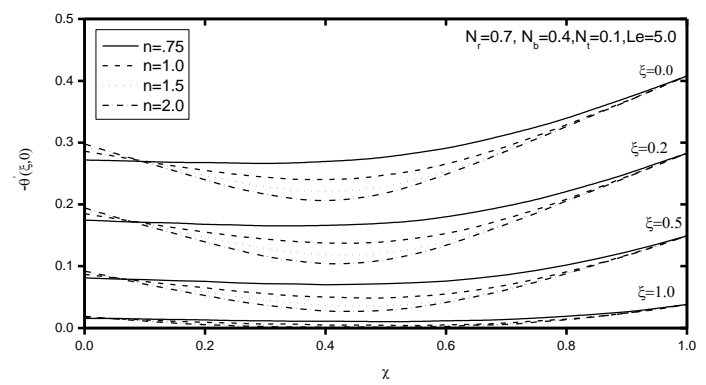

Fig. 2. Effects of $\mathrm{n}$ and $\xi$ on the local Nusselt number

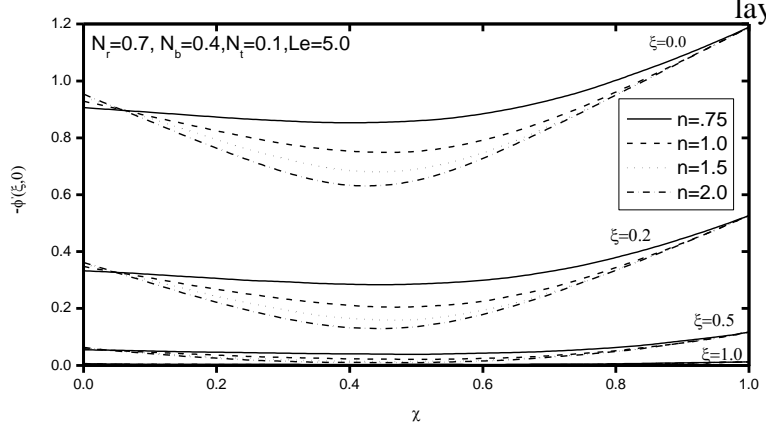

Fig. 3. Effects of $\mathrm{n}$ and $\xi$ on the local Sherwood number

Figure 2 and Fig. 3 show the variations of on the local Nusselt number $N u_{x}$, and the local Sherwood number $S h_{x}$, for different values of the power-law index $\mathrm{n}$ and the injection parameter $\xi$ in the entire range $0 \leq \chi \leq 1$, respectively. As seen from the definitions of $\mathrm{Nu}_{\mathrm{x}}$, and $\mathrm{Sh}_{\mathrm{x}}$, they are directly proportional to $-\theta^{\prime}(\xi, 0)$ and $-\phi^{\prime}(\xi, 0)$, respectively. For different values of the power-law index $n$ and the injection parameter $\xi$ in the entire range $0 \leq \chi \leq 1$, respectively. It is found that increasing the power-law index $\mathrm{n}$ leads to increase in both the local Nusselt or Sherwood numbers for smaller values of mixed convection parameter $\chi$ which indicate almost pure free convection regime and decreases the local Nusselt and Sherwood numbers for between 0 and 1 which correspond to the mixed convection regime whereas the local Nusselt and Sherwood numbers remain almost unchanged as the values of $\chi$ close to unity which indicate almost pure forced convection. On other hand as the values of the injection parameter $\xi$ increase both of heat transfer rate (local Nusselt number) and mass transfer rate (local Sherwood number) decrease in the entire range $0 \leq \chi \leq 1$.

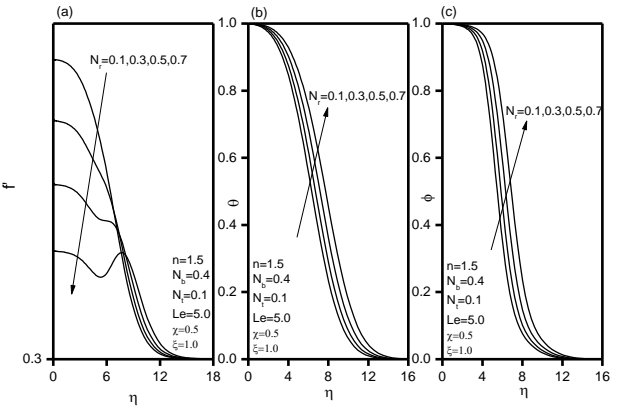

Fig. 4. Effects of $\mathrm{Nr}$ on the (a) velocity, (b) temperature, (c) volume fraction profiles

Figure 4(a) - 4(c) depict the effects of the buoyancy ratio $\mathrm{Nr}$ on the dimensionless velocity, temperature and volume fraction profiles. In general, increases in the value of $\mathrm{Nr}$ have the tendency to cause more induced flow along the plate surface. This behavior in the flow velocity is accompanied by decreases in the fluid temperature and volume fraction species as well as decreases in the thermal and volume fraction boundary layers as $\mathrm{N}$ increases from 0.1 to 0.7 .

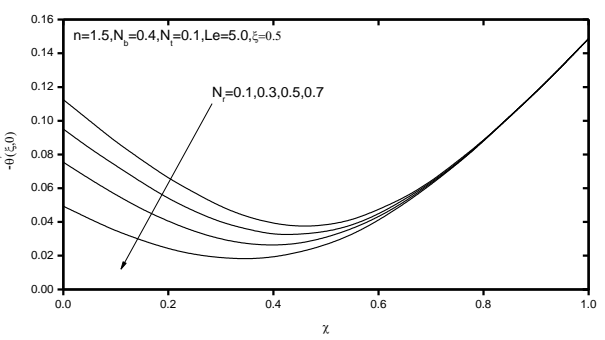

Fig. 5. Effects of $\mathrm{Nr}$ on the local Nusselt number

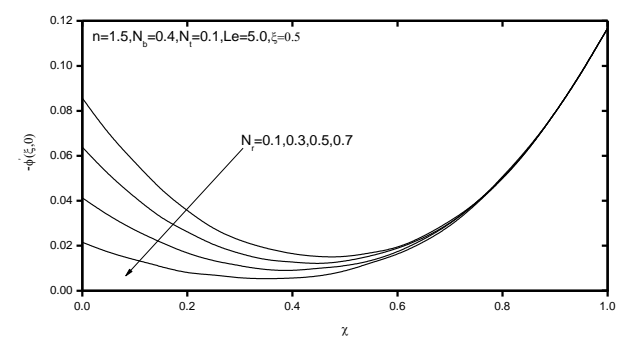

Fig. 6. Effects of $\mathrm{Nr}$ on the local Sherwood number

On other hand Fig. 5 and Fig. 6 illustrate the influence of the buoyancy ratio $\mathrm{Nr}$ on the local Nusselt number $N u_{x}$, and the local Sherwood - $\phi^{\prime}(\xi, 0)$, in the entire range $0 \leq \chi \leq 1$, The decreases in the fluid temperature and volume fraction as $\mathrm{Nr}$ increases mentioned above causes the negative values of the wall temperature and volume fraction slopes to increase yielding increases in both the local Nusselt and Sherwood numbers in the entire range of mixed convection parameter $0 \leq \chi \leq 1$. However, for $\chi=1$ (forced convection limit), the flow is uncoupled from the thermal and solutal buoyancy effects and therefore, the local Nusselt and Sherwood numbers are constant for all values of $\mathrm{Nr}$. The influence of the buoyancy force is significant for large values of $\chi$ 
but there is an apparent decrease in the maximum velocity inside the boundary layer. The reason for this trend is that for a vertical plate the buoyancy force acts normal to the forced flow. Also, from the definition of $\chi$, it is seen that increases in the value of the parameter $\mathrm{Ra}_{\mathrm{x}} / \mathrm{Pe}_{\mathrm{x}}$ causes the mixed convection parameter $\chi$ to decrease. Thus, small values of $\mathrm{Ra}_{x} / \mathrm{Pe}_{\mathrm{x}}$ correspond to values of $\chi$ close to unity which indicate almost pure forced convection regime On the other hand, high values of $\mathrm{Ra}_{\mathrm{x}} / \mathrm{Pe}_{\mathrm{x}}$ correspond to values of $\chi$ close to zero which indicate almost pure free convection regime. Furthermore, moderate values of $\mathrm{Ra}_{\mathrm{x}} / \mathrm{Pe}_{\mathrm{x}}$ represent values of $\chi$ between 0 and 1 which correspond to the mixed convection regime. For the forced convection limit $(\chi=1)$ it is clear from Eq. (8) that the velocity in the boundary layer $\mathrm{f}^{\prime}$ is uniform irregardless of the value of $n$. However, for smaller values of $\chi$ (higher values of $\mathrm{Ra}_{\mathrm{x}} / \mathrm{Pe}_{\mathrm{x}}$ ) at a fixed value of $\mathrm{Nr}$, the buoyancy effect increases. As this occurs, the fluid velocity close to the wall increases for values of $\chi<0.5$ due to the buoyancy effect which becomes maximum for $\chi=0$ (free convection limit) because the buoyancy force normal to the plate increases and acts to retard the flow in the streamwise direction within the boundary layer under a strong buoyant flow (small values of $\chi$ ). This decrease and increase in the fluid velocity $\mathrm{f}^{\prime}$ as $\chi$ is decreased from unity to zero is accompanied by a respective increase and a decrease in the fluid temperature and volume fraction. As a result, the local Nusselt and Sherwood numbers tend to decrease and then increase as $\chi$ is increased from 0 to unity forming slight dips.

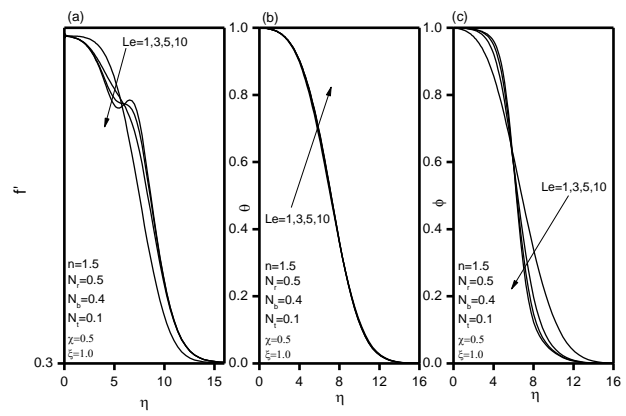

Fig. 7. Effects of Le on the (a) velocity,

(b) temperature, (c) volume fraction profiles

The velocity, temperature and volume fraction profiles for various values of Lewis number Le are shown in Figs. 7(a)-(c). It is observed that as Le increases the velocity continuously decreases with $\eta$ while the volume fraction increases but the opposite behavior far away from the plate. As expected, the velocity effects are more dominant near the wall. Also, the velocity decreases as Le increases in the vicinity of the plate but the reverse happens as one moves away from it. It is evident from Fig. 7(a) and Fig. 7(c) that both the velocity and volume fraction profiles satisfy the boundary conditions Eq. (11) a and Eq. (11) b. Thus, these figures support the validity of the present results. In addition, it is observed from Fig. (7) b that the temperature slight increases with an increase in Le and tends to zero at the edge of the boundary layer.
Figure 8 and Fig. 9 illustrate the effect of increasing the Lewis number Le on both of the local Nusselt number $N u_{x}$ and the local Sherwood number $S h_{x}$ in the entire range $0 \leq \chi \leq 1$, respectively. It can be seen that as the Lewis number Le increases, the resistance to flow and the wall slopes of the temperature and volume fraction profiles increase as shown earlier in Figs. 7(b)-7(c), this produces reductions in all the Nusselt and Sherwood numbers.

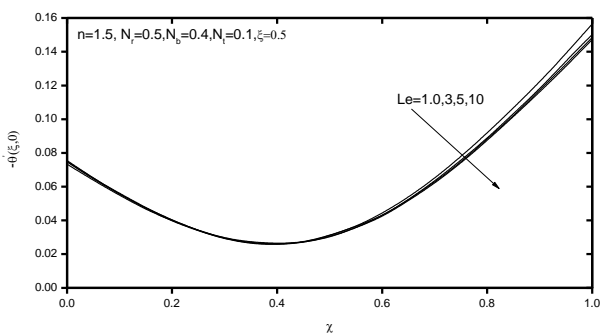

Fig. 8. Effects of Le on the local Nusselt number.

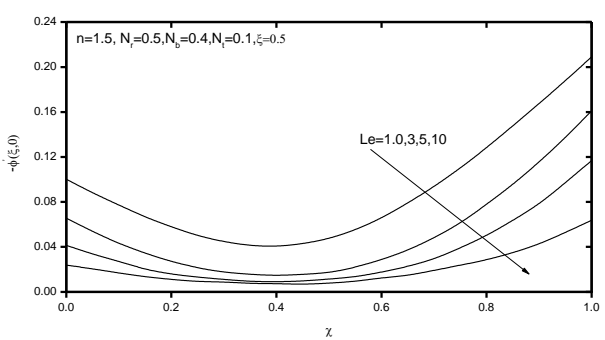

Fig. 9. Effects of Le on the local Sherwood number

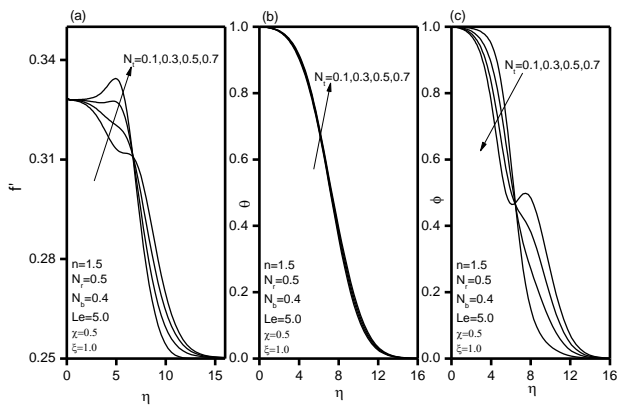

Fig. 10. Effects of Nt on the (a) velocity, (b) temperature, (c) volume fraction profiles

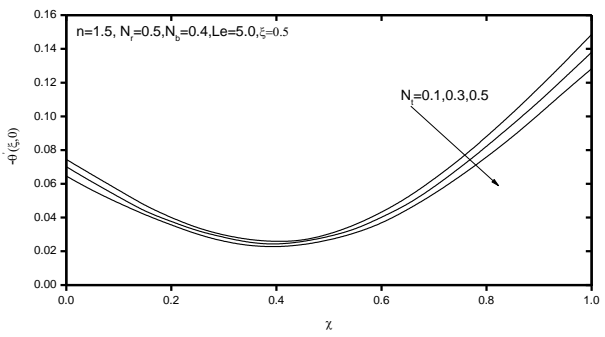

Fig. 11. Effects of Nt on the local Nusselt number 


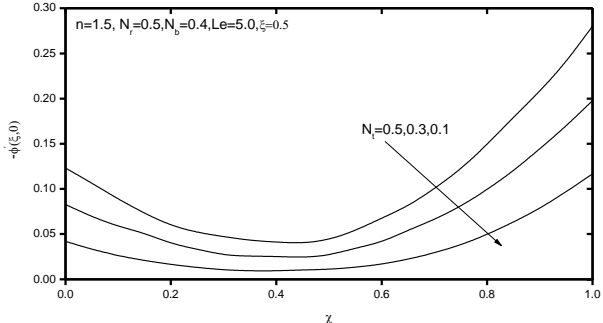

Fig. 12. Effects of Nt on the local Sherwood number

The effects of the thermophoresis parameter Nt on the velocity and temperature and volume fraction profiles are presented in Figs. 10(a)-10(c), respectively. It is observed that increasing the thermophoresis parameter $\mathrm{Nt}$ has a tendency to accelerate the flow and slight increases in the temperature profiles. This, in turn, produces increases in the maximum velocity and the fluid temperature and decreases in the volume fraction profiles. In addition, as seen from Fig. 11 and Fig. 12, increases in the value of the thermophoresis parameter $\mathrm{Nt}$ cause reductions in all of the local Nusselt and Sherwood numbers.

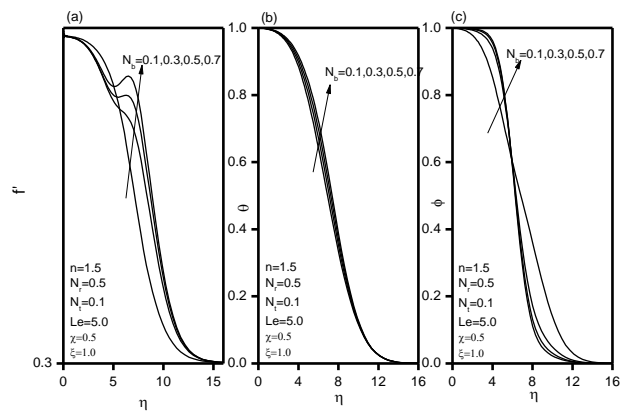

Fig. 13. Effects of $\mathrm{Nb}$ on the (a) velocity, (b) temperature, (c) volume fraction profiles

Figures 13(a)-13(c) present the changes in the velocity, temperature and volume fraction profiles for various values of the Brownian motion parameter $\mathrm{Nb}$, respectively. The effect of increasing the Brownian motion parameter $\mathrm{Nb}$ is limited to decreasing slightly the wall slope of the velocity with $\eta$ while the volume fraction increases but the opposite behavior far away from the plate. This decreasing the flow velocity at the wall is achieved at the expense of slight enhancement in the fluid temperature and volume fraction whereas a significant reduction in the concentration boundary layers as seen from Figs. 13(b)-13(c).

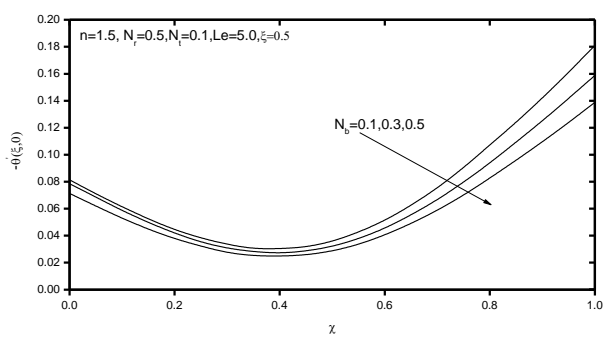

Fig. 14. Effects of $\mathrm{Nb}$ on the local Nusselt number

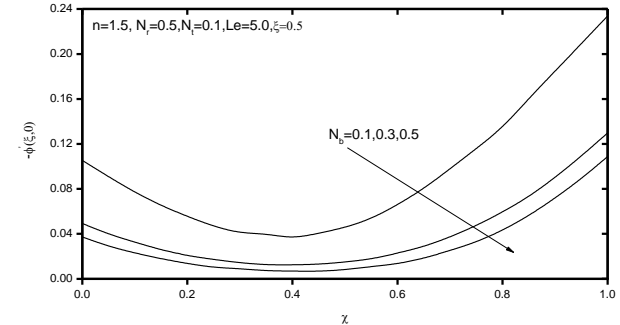

Fig. 15. Effects of $\mathrm{Nb}$ on the local Sherwood number

Figure 14 and Fig. 15 illustrate the effects of the Brownian motion parameter $\mathrm{Nb}$ on the local Nusselt number $N u_{x}$ and the local Sherwood number $S h_{x}$ in the range $0 \leq \chi \leq 1$, respectively. Obviously, increasing the Brownian motion parameter $\mathrm{Nb}$ decreases the flow along the plate surface while its temperature and volume fraction increase causing the negative wall slope of the temperature and volume fraction profiles to decrease. This yields reductions in all of the local Nusselt and Sherwood numbers.

\section{CONCLUSION}

The effects of Brownian motion and thermophoresis on the mixed convection boundary layer flow of nonNewtonian nanofluids past a preamble vertical flat plate embedded in a porous medium are considered. A mixed convection parameter for the entire range of freeforced-mixed convection is employed and a set of nonsimilar equations are obtained. These equations are solved numerically by an efficient implicit, iterative, finite-difference method. Comparisons with previously published work were performed and the results are found to be in excellent agreement. Numerical results for the velocity, temperature, and volume fraction profiles as well as the local Nusselt number and the local Sherwood number were reported graphically. It was found that both the local Nusselt and Sherwood numbers decreased due to increase in either of the power-law fluid index in the pure free convection regime and decreases the local Nusselt and Sherwood numbers in the mixed convection regime whereas the local Nusselt and Sherwood numbers remain almost unchanged as the values in the pure forced convection. However, they both decreased due to increases in either of the injection parameter. Also, It was found that as the buoyancy ratio increased, both the local Nusselt and Sherwood numbers increased in the entire range of free and mixed convection regime while they remained constant for the forced-convection regime. Finally, both the local Nusselt and Sherwood numbers decreased as either of Lewis number, Brownian motion parameter or thermophoresis effects increased. Moreover, it is shown that the effects of Lewis number, Brownian motion or thermophoresis parameters are more pronounced on the local Sherwood number than on the local Nusselt number.

\section{REFERENCES}

Abu-Nada, E. (2008). Application of nanofluids for heat transfer enhancement of separated flows encountered in a backward facing step. 
International Journal of Heat and Fluid Flow 29('1), 242-249.

Abu-Nada, E. and H. F. Oztop (2009). Effects of inclination angle on natural convection in enclosures filled with $\mathrm{Cu}$-water nanofluid. International Journal of Heat and Fluid Flow 30(4), 669-678.

Beithou, N., K. Albayrak and A. Abdulmajeed (1998). Effect of porosity on the free convection flow of non-Newtonian fluids along vertical plate embedded in a porous media. Turkish J. of Eng. Envir. Sci. 22(3), 203-209.

Blottner, F. G. (1970) Finite-difference methods of solution of the boundary-layer equations. AIAA Journal 8(2), 193-205.

Chamkha, A. J. (2010). Heat and mass transfer of a non-Newtonian fluid flow over a permeable wedge in porous media with variable wall temperature and concentration and heat source or sink. Wseas Transactions On Heat And Mass Transfer 5(1), 11-20.

Chamkha, A. J., A. M. Aly and H. Al-Mudhaf (2011). Laminar MHD mixed convection flow of a nanofluid along a stretching permeable surface in the presence of heat generation or absorption effects. International Journal of Microscale and Nanoscale Thermal and Fluid Transport Phenomena 2(1), 51-70

Chamkha, A. J., R. S. R. Gorla and K. Ghodeswar (2011). Non-similar solution for natural convective boundary layer flow over a sphere embedded in a porous medium saturated with a nanofluid. Transport in Porous Media 86(1), 13-22

Chen H. T. and C. K. Chen (1988). Free convection of non-Newtonian fluids along a vertical plate embedded in a porous medium. Journal of Heat Transfer 110(1), 257-60.

Chiu, T. W., R. J. Wakeman, P.R. Harris and O.F.J. Meuric (1996). Effect of non-Newtonian fluids and porous media parameters on two-phase flow in porous media. Part A: Transactions of the Instit. Of Chem. Engineers 74(2), 220-231.

Choi, S. U. S. (1995). Enhancing thermal conductivity of fluids with nanoparticle. in: D A. Siginer, H.P. Wang (Eds.), Developments and Applications of Non-Newtonian Flows, ASME FED, 231/MD (66), 99-105.
Duangthongsuk, W. and S. Wongwises (2008). Effect of thermophysical properties models on the predicting of the convective heat transfer coefficient for low concentration nanofluid. International Communications in Heat and Mass Transfer 35(10), 1320-1326.

EL-Kabeir, S. M. M., A. J. Chamkha and A. M. Rashad (2010). Heat and Mass Transfer by MHD Stagnation-Point Flow of a Power-Law Fluid towards a Stretching Surface with Radiation, Chemical Reaction and Soret and Dufour Effects. International Journal of Chemical Reactor Engineering 8(1), 1-11.

Gorla, R. S. R. and M. Kumari (2000). Nonsimilar solutions for mixed convection in non-Newtonian fluids along a wedge with variable surface heat flux in a porous medium. J. Porous Media 3(2), 181-184.

Gorla, R. S. R., A. Slaouti and H.S. Takhar (1997). Mixed convection in non-Newtonian fluids along a vertical plate in porous media with surface mass transfer. International Journal of Numerical Methods for Heat and Fluid Flow 7(6), 598-608.

Hwang, K. S., J. H. Lee and S. P. Jang (2007). Buoyancy-driven heat transfer of water-based $\mathrm{Al}$ nanofluids in a rectangular cavity. International Journal of Heat and Mass Transfer 50(19-20), 4003-4010.

Jou, R.Y. and S. C. Tzeng (2006). Numerical research of nature convective heat transfer enhancement filled with nanofluids in rectangular enclosures. International Communications in Heat and Mass Transfer 33(6), 727-736.

Kang, H. U., S. H. Kim and J. M. Oh (2006). Estimation of thermal conductivity of nanofluid using experimental effective particle volume. Experimental Heat Transfer 19(3), 181-191.

Kumar, S., S. K. Prasad and J. Banerjee (2009). Analysis of flow and thermal field in nanofluid using a single phase thermal dispersion model. Applied Mathematical Modeling 34(3), 573-592

Maiga, S. E., S. J. Palm, C. T. Nguyen, G. Roy and N. Galanis (2005). Heat transfer enhancement by using nanofluids in forced convection flows. International Journal of Heat and Fluid Flow 26(4), 530-546. 
A. M. Rashad et al. / JAFM, Vol. 6, No. 2, pp. 301-309, 2013.

Nakayama A. and H. Koyama (1991). Buoyancy induced flow of non-Newtonian fluids over a nonisothermal body of arbitrary shape in a fluidsaturated porous medium. Applied Scientific Research 48(1), 55-70.

Oztop, H. F. and E. Abu-Nada (2008). Numerical study of natural convection in partially heated rectangular enclosures filled with nanofluids. International Journal of Heat and Mass Transfer 29(5), 1326-1336.

Pascal, J. P. and H. Pascal (1997). Free convection in a non-Newtonian fluid saturated porous media with lateral mass flux. International Journal of NonLinear Mechanics 32(3), 471-482.

Tiwari, R. K. and M. K. Das (2007). Heat transfer augmentation in a two-sided lid-driven differentially heated square cavity utilizing nanofluids. International Journal of Heat and Mass Transfer 50(9-10), 2002-2018.
Xuan, Y. and W. Roetzel (2000). Conceptions for heat transfer correlation of nanofluids. International Journal of Heat and Mass Transfer 43(19), 37013707.

Yang, Y. T. and S. J. Wang (1996). Free convection heat transfer of non-Newtonian fluids over axisymmetric and two dimensional bodies of arbitrary shape embedded in a fluid-saturated porous media. International Journal of Heat and Mass Transfer 39(1), 203-210.

Yih, K. A. (1998). Uniform lateral mass flux effect on natural convection of non-Newtonian fluids over a cone in porous media. International Communications in Heat and Mass Transfer 25(7), 959-968. 